EUROPEAN JOURNAL OF PURE AND APPLIED MATHEMATICS

Vol. 14, No. 4, 2021, 1490-1506

ISSN 1307-5543 - ejpam.com

Published by New York Business Global

\title{
On Connected Partial Domination in Graphs
}

\author{
Jessa Mae C. Cabulao ${ }^{1, *}$, Rowena T. Isla ${ }^{1,2}$ \\ ${ }^{1}$ Department of Mathematics and Statistics, College of Science and Mathematics, \\ Mindanao State University-Iligan Institute of Technology, 9200 Iligan City, Philippines \\ ${ }^{2}$ Center for Graph Theory, Algebra, and Analysis, Premier Research Institute of Science \\ and Mathematics, Mindanao State University-Iligan Institute of Technology, 9200 \\ Iligan City, Philippines
}

\begin{abstract}
This paper introduces and investigates a variant of partial domination called the connected $\alpha$-partial domination. For any graph $G=(V(G), E(G))$ and $\alpha \in(0,1]$, a set $S \subseteq V(G)$ is an $\alpha$-partial dominating set in $G$ if $|N[S]| \geq \alpha|V(G)|$. An $\alpha$-partial dominating set $S \subseteq V(G)$ is a connected $\alpha$-partial dominating set in $G$ if $\langle S\rangle$, the subgraph induced by $S$, is connected. The connected $\alpha$-partial domination number of $G$, denoted by $\partial_{C \alpha}(G)$, is the smallest cardinality of a connected $\alpha$-partial dominating set in $G$. In this paper, we characterize the connected $\alpha$-partial dominating sets in the join and lexicographic product of graphs for any $\alpha \in(0,1]$ and determine the corresponding connected $\alpha$-partial domination numbers of graphs resulting from the said binary operations. Moreover, we establish sharp bounds for the connected $\alpha$-partial domination numbers of the corona and Cartesian product of graphs. Furthermore, we determine $\partial_{C \alpha}(G)$ of some special graphs when $\alpha=\frac{1}{2}$. Several realization problems are also generated in this paper.
\end{abstract}

2020 Mathematics Subject Classifications: 05C69

Key Words and Phrases: Partial domination, Connected Partial domination, Join, Corona, Lexicographic product, Cartesian product

\section{Introduction}

The study of domination has been an extensively investigated subject in graph theory. Specifically, many research studies have been made concerning the concepts of "dominating set" and "domination number" which were first introduced by Oystein Ore in 1962 [1]. A dominating set $D \subseteq V(G)$ for a graph $G=(V(G), E(G))$ is a set where every vertex $u \in V(G) \backslash D$ is adjacent to some vertex $v \in D$ such that $u v \in E(G)$. The domination number of $G$, denoted by $\gamma(G)$, is the minimum cardinality of a dominating set in $G$. A $\gamma$-set in $G$ is any dominating set in $G$ of cardinality $\gamma(G)$. We refer the reader to T. Haynes

* Corresponding author.

DOI: https://doi.org/10.29020/nybg.ejpam.v14i4.4168

Email addresses: jessamae.cabulao@g.msuiit.edu.ph (J.M. Cabulao),

rowena.isla@g.msuitt.edu.ph (R. Isla) 
et al. [6] for a comprehensive treatment of the theoretical, algorithmic, and application aspects of domination in graphs, as well as to varieties of domination.

The connected domination in graphs was first defined by E. Sampathkumarin and H.B. Walikar in 1979 [12]. A connected dominating set $D \subseteq V(G)$ for a graph $G=(V(G), E(G))$ is a dominating set where the subgraph $\langle D\rangle$ induced by the set $D$ is connected. The connected domination number of $G$, denoted by $\gamma_{c}(G)$, is the minimum cardinality of a connected dominating set in $G$. A $\gamma_{c^{-}}$set in $G$ is any dominating set in $G$ of cardinality $\gamma_{c}(G)[11]$.

In 2009, W. Duckworth and B. Mans [4] presented several algorithms for finding small connected dominating sets of regular graphs. In 2012, H. Karami et al. [8] determined sharp bounds on the sum of the connected domination number of a graph and its complement. One of the many important applications of connected domination is in wireless networks. In order to achieve scalability and efficiency in the routing of messages in mobile ad hoc networks (MANETs), new algorithms have emerged that rely on a virtual network infrastructure, which organizes ordinary nodes into a hierarchy. The construction of this infrastructure is the primary application of connected dominating sets (CDSs) in wireless networks [13]. A research work in 2017 by S. Kaspar et al. [5] that utilizes connected domination has resulted out of the real life application of improving an existing facility location network into a stronger and more reliable one.

It can be observed that we can determine a dominating set $S$ if the closed neighborhood $N[S]$ is equal to the vertex set $V(G)$ of $G$. However, there may be situations or instances where providing a complete coverage of a network will not be necessary. Thus, a new notion called partial domination in graphs was developed. In this case, it will suffice to dominate only a certain number of nodes in a network $[2,9]$.

In 2017, both Case et al. [9] and Das [3] independently studied $\alpha$-partial domination in graphs and later on published their respective papers on the subject. Das worked on the general value of $\alpha$ with $0<\alpha \leq 1$, whereas Case et al. primarily focused on $\alpha=\frac{1}{2}$ where the sets of vertices dominate only at least half of the vertex set of a graph. In the same year, Y. Caro and A. Hansberg [2] proved that if $G$ is a connected graph on $n \geq 6$ vertices, then there exists a set of vertices $D$ with $|D| \leq \frac{n}{3}$ and such that $V(G) \backslash N[D]$ is an independent set, a result in the direction of partial domination with constrained structure on the graph induced by the non-dominated vertices.

Let $\alpha \in(0,1]$. An $\alpha$-partial dominating set $S \subseteq V(G)$ for a graph $G=(V(G), E(G))$ is a set where $|N[S]| \geq \alpha|V(G)|$. The $\alpha$-partial domination number of $G$ is the minimum cardinality of an $\alpha$-partial dominating set in $G$, and is denoted by $\partial_{\alpha}(G)$. An $\partial_{\alpha}$-set in $G$ is any partial dominating set in $G$ of cardinality $\partial_{\alpha}(G)$.

In 2019, R. Macapodi, R. Isla and S. Canoy [7] characterized the partial dominating sets in the join, corona, lexicographic and Cartesian products of graphs and determined the exact values or sharp bounds of the corresponding partial domination number of the said graphs. In the same year, R. Macapodi and R. Isla [10] published another paper where they characterized the total partial dominating sets in the join, corona, lexicographic product and Cartesian product of graphs. They also determined the exact values or sharp bounds of the corresponding total partial domination number of these graphs. 
This paper combines the concepts of connected domination and partial domination in graphs. Let $G$ be a graph and $\alpha \in(0,1]$. An $\alpha$-partial dominating set $S \subseteq V(G)$ is a connected $\alpha$-partial dominating set in $G$ if the subgraph $\langle S\rangle$ induced by $S$, is connected. The connected $\alpha$-partial domination number of $G$, denoted by $\partial_{C \alpha}(G)$, is the smallest cardinality of a connected $\alpha$-partial dominating set in $G$. A connected $\alpha$-partial dominating set $S$ in $G$ with $|S|=\partial_{C \alpha}(G)$ is referred to as a $\partial_{C \alpha^{-}}$set in $G$.

The join $G+H$ of two graphs $G$ and $H$ is the graph with vertex-set $V(G+H)=$ $V(G) \cup V(H)$ and edge-set $E(G+H)=E(G) \cup E(H) \cup\{u v: u \in V(G), v \in V(H)\}$. The corona of two graphs $G$ and $H$, denoted by $G \circ H$, is the graph obtained by taking one copy of $G$ of order $n$ and $n$ copies of $H$, and then joining the $i$-th vertex of $G$ to every vertex in the $i$-th copy of $H$. For every $v \in V(G)$, we denote by $H^{v}$ the copy of $H$ whose vertices are joined or attached to the vertex $v$. For each $v \in V(G)$, the subgraph $\langle v\rangle+H^{v}$ of $G \circ H$ will be denoted by $v+H^{v}$. The lexicographic product (composition) of two graphs $G$ and $H$, denoted by $G[H]$, is the graph with vertex-set $V(G[H])=V(G) \times V(H)$ and edge-set $E(G[H])$ satisfying the following conditions: $\left(u_{1}, v_{1}\right)\left(u_{2}, v_{2}\right) \in E(G[H])$ if and only if either $u_{1} u_{2} \in E(G)$ or $u_{1}=u_{2}$ and $v_{1} v_{2} \in E(H)$. The Cartesian product of two graphs $G$ and $H$, denoted by $G \square H$, is the graph with vertex-set $V(G \square H)=V(G) \times V(H)$ and edge-set $E(G \square H)$ satisfying the following conditions: $\left(u_{1}, v_{1}\right)\left(u_{2}, v_{2}\right) \in E(G \square H)$ if and only if either $u_{1} u_{2} \in E(G)$ and $v_{1}=v_{2}$ or $u_{1}=u_{2}$ and $v_{1} v_{2} \in E(H)$.

In this paper, we characterize the connected $\alpha$-partial dominating sets in the join and lexicographic product of graphs for any $\alpha \in(0,1]$ and determine the corresponding connected $\alpha$-partial domination numbers of graphs resulting from the said binary operations. Moreover, we establish sharp bounds for the connected alpha-partial domination numbers of the corona and Cartesian product of graphs. Furthermore, we also determine $\partial_{C \alpha}(G)$ of some special graphs when $\alpha=\frac{1}{2}$. Several realization problems are also generated in this paper.

\section{Preliminaries}

This section gives some preliminary results, the connected partial domination number of some special graphs, some realization problems involving the partial domination number and the connected partial domination number, and some concepts and known results on partial domination that will be used to characterize connected partial dominating sets in the join of graphs.

The first remark immediately follows from the definitions of the concepts involved.

Remark 1. Every connected $\alpha$-partial dominating set is an $\alpha$-partial dominating set.

The next remark immediately follows from Remark 1.

Remark 2. Let $G$ be a nontrivial connected graph and let $\alpha \in(0,1]$. Then $\partial_{C \alpha}(G) \geq$ $\partial_{\alpha}(G)$. 
Remark 3. The bound given in Remark 2 is sharp. However, the inequality can be attained.

To see this, consider the graph $G_{1}$ in Figure 1 . Let $\alpha=\frac{1}{2}$. It can be verified that $S_{1}=\{d, k\}$ is a $\partial_{\alpha}$-set and $S_{2}=\{d, e, f, g\}$ is a $\partial_{C \alpha}$-set in $G_{1}$. Thus, $\partial_{C \alpha}\left(G_{1}\right)=4>2=$ $\partial_{\alpha}\left(G_{1}\right)$.

Next, consider the graph $G_{2}$ in Figure 2 and let $\alpha=\frac{2}{3}$. It can be verified that $S_{3}=\{b, c\}$ is both a $\partial_{\alpha}$-set and a $\partial_{C \alpha}$-set in $G_{2}$. Thus, $\partial_{\alpha}\left(G_{2}\right)=\partial_{C \alpha}\left(G_{2}\right)=2$.

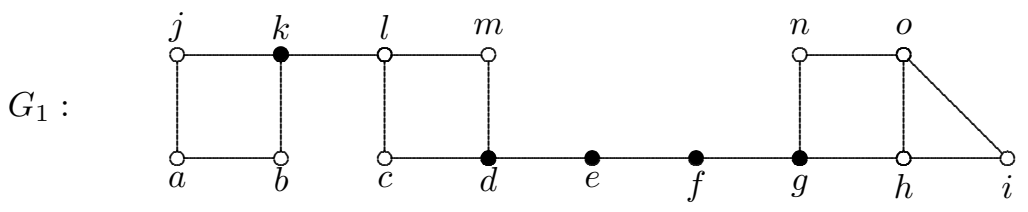

Figure 1: A graph $G_{1}$ with $\partial_{C \alpha}\left(G_{1}\right)=4>2=\partial_{\alpha}\left(G_{1}\right)$

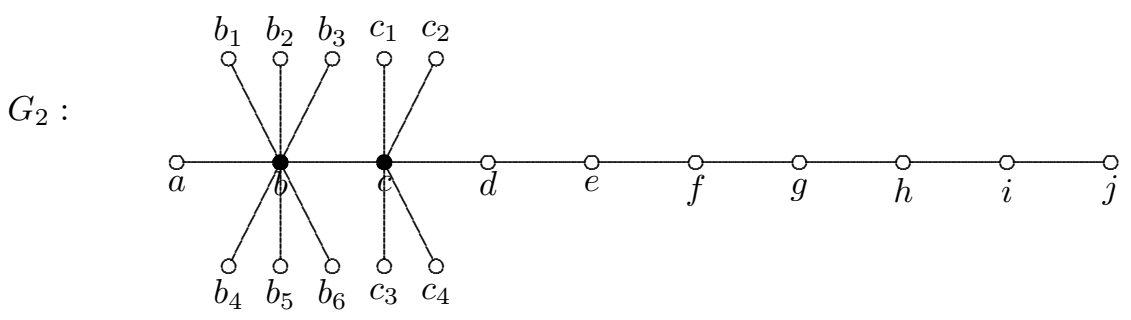

Figure 2: A graph $G_{2}$ with $\partial_{C \alpha}\left(G_{2}\right)=\partial_{\alpha}\left(G_{2}\right)=2$, where $\alpha=\frac{2}{3}$

Remark 4. Let $G$ be a nontrivial connected graph and let $\alpha, \beta \in(0,1]$. If $\alpha<\beta$, then $\partial_{\alpha}(G) \leq \partial_{\beta}(G)$. Moreover, $\partial_{C \alpha}(G) \leq \partial_{C \beta}(G)$.

Remark 5. Let $G$ be a nontrivial connected graph and let $\alpha \in(0,1]$. Then,

(i) $1 \leq \partial_{C \alpha}(G) \leq \gamma(G) \leq \gamma_{c}(G)$, and

(ii) if $\gamma(G)=1$, then $\partial_{C \alpha}(G)=1$.

The next result immediately follows from Remark 5(ii) and from $\gamma(G)=1$ for $G \in$ $\left\{K_{n}, F_{n}, K_{1, n}, W_{n}\right\}$.

Proposition 1. Let $n$ be a positive integer and let $\alpha \in(0,1]$. Let $G$ be $K_{n}, F_{n}$ or $K_{1, n}$. Then $\partial_{C \alpha}(G)=1$ for $n \geq 1$. Moreover, $\partial_{C \alpha}\left(W_{n}\right)=1$ for $n \geq 3$.

Proposition 2. For any complete bipartite graph $K_{m, n}$, where $m \leq n, \partial_{C \alpha}\left(K_{m, n}\right)=1$ if and only if $\alpha \leq \frac{n+1}{m+n}$. Otherwise, $\partial_{C \alpha}\left(K_{m, n}\right)=2$.

Proof: Denote the vertices in the partite set with $m$ vertices by $u_{1}, u_{2}, \ldots, u_{m}$ and the vertices in the partite set with $n$ vertices by $v_{1}, v_{2}, \ldots, v_{n}$. Assume that $\partial_{C \alpha}\left(K_{m, n}\right)=1$. Let 
$S=\{u\}$ be a $\partial_{C \alpha}$-set of $K_{m, n}$. If $u=u_{k}$ for some $k$, then $\left|N_{K_{m}, n}[u]\right|=n+1 \geq \alpha(m+n)$, showing $\alpha \leq \frac{n+1}{m+n}$. Suppose that $u=v_{k}$ for some $k$. Then $\left|N_{K_{m, n}}[u]\right|=m+1 \geq \alpha(m+n)$. Since $m \leq n, n+1 \geq \alpha(m+n)$, showing the desired inequality.

Conversely, suppose that $\alpha \leq \frac{n+1}{m+n}$. Then $\alpha(m+n) \leq n+1$. For all $i=1,2, \ldots, m$, $\left|N_{G}\left[u_{i}\right]\right|=n+1 \geq \alpha(m+n)=\alpha\left|V\left(K_{m, n}\right)\right|$. Then, $S=\left\{u_{i}\right\}$ is a $\partial_{C \alpha}$-set of $K_{m, n}$ for all $i=1,2, \ldots, m$. Accordingly, $\partial_{C \alpha}\left(K_{m, n}\right)=1$. For $\frac{n+1}{m+n}<\alpha \leq 1, R=\left\{u_{i}, v_{j}\right\}$ for some $1 \leq i \leq m, 1 \leq j \leq n$ is clearly a $\partial_{C \alpha}$-set, hence $\partial_{C \alpha}\left(K_{m, n}\right)=2$.

Proposition 3. For any path or cycle of length $n, \partial_{C \frac{1}{2}}\left(P_{n}\right)=1$ for $1 \leq n \leq 4, \partial_{C \frac{1}{2}}\left(C_{n}\right)=$ 1 for $3 \leq n \leq 4$, and $\partial_{C \frac{1}{2}}\left(P_{n}\right)=\partial_{C \frac{1}{2}}\left(C_{n}\right)=\left\lceil\frac{n}{2}\right\rceil-2$ for $n \geq 5$.

Proof: Let $P_{n}=\left[v_{1}, v_{2}, \ldots, v_{n}\right]$. If $1 \leq n \leq 4$, then clearly, $\partial_{C \frac{1}{2}}\left(P_{n}\right)=1$. Suppose that $n \geq 5$. Let $S=\left\{u_{2}, \ldots, u_{\left\lceil\frac{n}{2}\right\rceil-1}\right\}$. Then $\langle S\rangle$ is connected and $\left|N_{P_{n}}[S]\right|=|S|+2=$ $\left(\left\lceil\frac{n}{2}\right\rceil-2\right)+2=\left\lceil\frac{n}{2}\right\rceil \geq \frac{1}{2} \cdot n$, hence $S$ is a connected $\frac{1}{2}$-partial dominating set in $P_{n}$. Thus, $\partial_{C \frac{1}{2}}\left(P_{n}\right) \leq|S|=\left\lceil\frac{n}{2}\right\rceil-2$. Moreover, any connected $\frac{1}{2}$-partial dominating set in $P_{n}$ should dominate at least $\left\lceil\frac{n}{2}\right\rceil$ of its vertices, thus it should consist of at least $\left\lceil\frac{n}{2}\right\rceil-2$ adjacent vertices, none of which is an end-vertex of $P_{n}$. Thus, $\partial_{C \frac{1}{2}}\left(P_{n}\right) \geq\left\lceil\frac{n}{2}\right\rceil-2$. Therefore, $\partial_{C \frac{1}{2}}\left(P_{n}\right)=\left\lceil\frac{n}{2}\right\rceil-2$.

Similarly, it can be shown that $\partial_{C \frac{1}{2}}\left(C_{n}\right)=\left\lceil\frac{n}{2}\right\rceil-2$ for $n \geq 5$, where a connected $\frac{1}{2}$-partial dominating set consists of at least $\left\lceil\frac{n}{2}\right\rceil-2$ adjacent vertices of $C_{n}$. Clearly, $\partial_{C \frac{1}{2}}\left(C_{3}\right)=\partial_{C \frac{1}{2}}\left(C_{4}\right)=1$.

We now present some realization problems.

Theorem 1. Let $a$ and $b$ be positive integers such that $b=3 a-2$. Then there exists $a$ connected graph $G$ such that $\partial_{\frac{1}{2}}(G)=a$ and $\partial_{C \frac{1}{2}}(G)=b$.

Proof: Case 1. $a=1$

Then $b=1$. Let $G$ be the graph shown in Figure 3. Now, $S=\left\{x_{1}\right\}$ is both a $\partial_{\frac{1}{2}}$-set and a $\partial_{C \frac{1}{2}}$-set of $G$. Hence, $\partial_{\frac{1}{2}}(G)=\partial_{C \frac{1}{2}}(G)=1$.

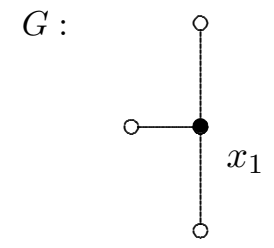

Figure 3: A graph $G$ with $\partial_{\frac{1}{2}}(G)=\partial_{C \frac{1}{2}}(G)=1$

Case 2. $a \geq 2$

Let $G$ be the graph shown in Figure 4. Put $A=\left\{x_{i}: i=1,2, \ldots, a\right\}$ and $B=$ $A \cup\left\{u_{k}: k=1,2, \ldots, 2 a-2\right\}$. Now, $|V(G)|=2 a+2(2 a-1)+1+2(2 a)=10 a-1$, $\left|N_{G}[A]\right|=|A|+(2 a-2)+2|A|+2=5 a$ and $\left|N_{G}[B]\right|=a+(2 a-2)+2 a+2=5 a$ so that 
$\left|N_{G}[A]\right|=\left|N_{G}[B]\right| \geq \frac{1}{2}|V(G)|=\frac{1}{2}(10 a-1)=5 a-\frac{1}{2}$. Clearly, $A$ and $B$ are $\partial_{\frac{1}{2}}$-set and $\partial_{C \frac{1}{2}^{-}}$ set in $G$, respectively. Thus, $\partial_{\frac{1}{2}}(G)=|A|=a$ and $\partial_{C \frac{1}{2}}(G)=|B|=a+(2 a-2)=3 a-2=b$.

$G:$

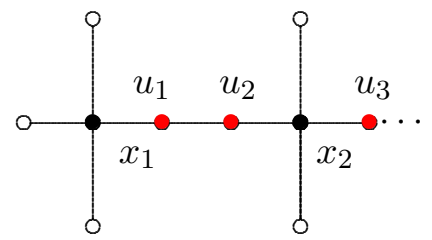

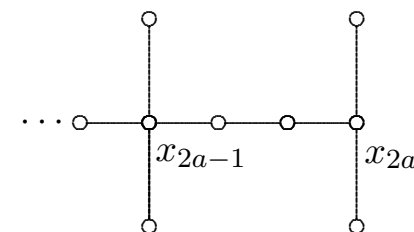

Figure 4: A graph $G$ with $\partial_{\frac{1}{2}}(G)=a<\partial_{C \frac{1}{2}}(G)=3 a-2=b$, where $a \geq 2$

This proves the assertion.

The next result immediately follows from Theorem 1 .

Corollary 1. Given a positive integer $m>1$ and $\alpha=\frac{1}{2}$, there exists a connected graph $G$ such that $\partial_{C \alpha}(G)-\partial_{\alpha}(G)=2 m-2$, that is, the difference $\partial_{C \alpha}-\partial_{\alpha}$ can be made arbitrarily large.

Proof: Let $a=m$. By Theorem 1, there exists a connected graph $G$ such that $\partial_{C \alpha}(G)-$ $\partial_{\alpha}(G)=(3 a-2)-a=2 m-2$.

Theorem 2. Given any positive integer $n \geq 2$, there exists a connected graph $G$ of order $5 n$ such that $\partial_{\alpha}(G)=\lceil\alpha n\rceil$ and $\partial_{C \alpha}(G)=3 \partial_{\alpha}(G)-2$ for $\alpha \in(0,1]$.

Proof:

Let $G$ be the graph shown in Figure 5. Then, the set $A=\left\{x_{j}: j=1,2, \ldots,\lceil\alpha n\rceil\right\}$ is a $\partial_{\alpha}$-set in $G$ and $B=A \cup\left\{u_{k}: k=1,2, \ldots, 2(\lceil\alpha n\rceil-1)\right\}$ is a $\partial_{C \alpha}$-set in $G$. Hence, $\partial_{\alpha}(G)=\lceil\alpha n\rceil$ and $\partial_{C \alpha}(G)=\lceil\alpha n\rceil+2(\lceil\alpha n\rceil-1)=\partial_{\alpha}(G)+2\left(\partial_{\alpha}(G)-1\right)=3 \partial_{\alpha}(G)-2$.

$G:$

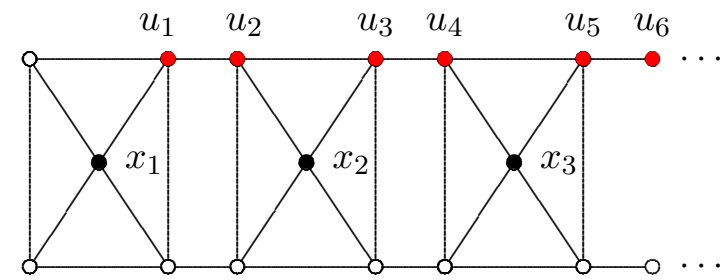

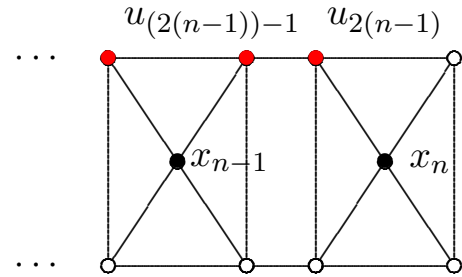

Figure 5: A graph $G$ with $\partial_{\alpha}(G)=a<\partial_{C \alpha}=3 a-2$

This proves the assertion.

The following concept is introduced in R. Macapodi et al. [7] and is used to characterize partial dominating sets in the join of graphs. 
Definition 1. [7] Let $G$ be a simple graph. Let $\alpha \in(0,1]$ and $k \in(-\infty, 0]$. A nonempty set $S \subseteq V(G)$ is an $(\alpha, k)$-partial dominating set in $G$ if $|N[S]| \geq \alpha|V(G)|+k$. The $(\alpha, k)$-partial domination number of $G$, denoted by $\partial_{\alpha, k}(G)$, is given by $\partial_{\alpha, k}(G)=$ $\min \{|S|: S$ is an $(\alpha, k)$-partial dominating set in $G\}$. Any $(\alpha, k)$-partial dominating set in $G$ with cardinality $\partial_{\alpha, k}(G)$ is referred to as an $\partial_{\alpha, k}$ set in $G$.

Remark 6. [7] Every $\alpha$-partial dominating set is an $(\alpha, k)$-partial dominating set. Consequently, $\partial_{\alpha, k}(G) \leq \partial_{\alpha}(G)$ for each $k \in(-\infty, 0]$.

Theorem 3. [7] Let $G$ and $H$ be connected graphs of orders $m$ and $n$, respectively, and let $\alpha \in(0,1]$. Then $C \subseteq V(G+H)$ is an $\alpha$-partial dominating set in $G+H$ if and only if at least one of the following is true:

(i) $C \subseteq V(G)$ and $C$ is an $(\alpha,(\alpha-1) n)$-partial dominating set in $G$.

(ii) $C \subseteq V(H)$ and $C$ is an $(\alpha,(\alpha-1) m)$-partial dominating set in $H$.

(iii) $C \cap V(G) \neq \varnothing$ and $C \cap V(H) \neq \varnothing$.

\section{Main Results}

This section presents characterizations of the connected $\alpha$-partial dominating sets in the join and lexicographic product for any $\alpha \in(0,1]$ and determines the corresponding connected $\alpha$-partial domination numbers of graphs resulting from the said binary operations. Moreover, some $\alpha$-partial dominating sets in the corona and Cartesian product of graphs are given and sharp bounds for their corresponding connected $\alpha$-partial domination numbers are established.

We first introduce the following concept which will be used to characterize the connected $\alpha$-partial dominating sets in the join of graphs.

Definition 2. Let $G$ be a connected graph. Let $\alpha \in(0,1]$ and $k \in(-\infty, 0]$. A nonempty set $S \subseteq V(G)$ is a connected $(\alpha, k)$-partial dominating set in $G$ if $|N[S]| \geq \alpha|V(G)|+k$ and $\langle S\rangle$, the subgraph induced by $S$, is connected . The connected $(\alpha, k)$-partial domination number of $G$, denoted by $\partial_{\alpha, k}^{C}(G)$, is given by

$$
\partial_{\alpha, k}^{C}(G)=\min \{|S|: S \text { is a connected }(\alpha, k) \text {-partial dominating set in } G\} \text {. }
$$

Any connected $(\alpha, k)$-partial dominating set in $G$ with cardinality $\partial_{\alpha, k}^{C}(G)$ is referred to as a $\partial_{\alpha, k}^{C}$-set in $G$.

Example 1. Consider the path of order 12 shown in Figure 6 . Clearly, the set $S_{1}=$ $\{2,3,4,5,6,7,8,9,10,11\}$ is a connected $(1,0)$-partial dominating set. For $k \in(-\infty, 0]$ and $\alpha=\frac{1}{2}$, the sets $S_{2}=\{2,3,4,5\}$ and $S_{3}=\{7,8,9,10,11\}$ are $\left(\frac{1}{2}, k\right)$-partial dominating sets in $P_{12}$. It can be observed that $S_{1}$ is a $\partial_{1,0}^{C}$-set while $S_{2}$ is a $\partial_{\frac{1}{2}, k}^{C}$-set in $P_{12}$. Hence, $\partial_{1,0}^{C}\left(P_{12}\right)=10$ and $\partial_{\frac{1}{2}, k}^{C}\left(P_{12}\right)=4$. 


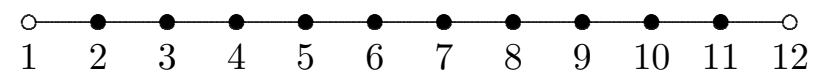

Figure 6: The path $P_{12}$ with $\partial_{1,0}^{C}\left(P_{12}\right)=10$ and $\partial_{\frac{1}{2}, k}^{C}\left(P_{12}\right)=4$

Remark 7. Every connected $(\alpha, k)$-partial dominating set is an $(\alpha, k)$-partial dominating set, where $\alpha \in(0,1]$ and $k \in(-\infty, 0]$.

Theorem 4. Let $G$ and $H$ be connected graphs of orders $m$ and $n$, respectively, and let $\alpha \in(0,1]$. Then $C \subseteq V(G+H)$ is a connected $\alpha$-partial dominating set in $G+H$ if and only if at least one of the following is true:

(a) $C \subseteq V(G)$ and $C$ is a connected $(\alpha,(\alpha-1) n)$-partial dominating set in $G$.

(b) $C \subseteq V(H)$ and $C$ is a connected $(\alpha,(\alpha-1) m)$-partial dominating set in $H$.

(c) $C \cap V(G) \neq \varnothing$ and $C \cap V(H) \neq \varnothing$.

Proof: Suppose that $C \subseteq V(G+H)$ is a connected $\alpha$-partial dominating set in $G+H$. Then, by Remark 1, $C$ is an $\alpha$-partial dominating set in $G+H$. By Theorem 3, at least one of the following is true: $(i) C \subseteq V(G)$ and $C$ is an $(\alpha,(\alpha-1) n)$-partial dominating set in $G,(i i) C \subseteq V(H)$ and $C$ is an $(\alpha,(\alpha-1) m)$-partial dominating set in $H$, or (iii) $C \cap V(G) \neq \varnothing$ or $C \cap V(H) \neq \varnothing$. Suppose that $(i)$ holds. Since $C$ is a connected $\alpha$ partial dominating set in $G+H$ and $C \subseteq V(G)$, it follows that $C$ is a connected $\alpha$-partial dominating set in $G$. Moreover, $\left|N_{G+H}[C]\right|=\left|N_{G}[C]\right|+n \geq \alpha m+n \geq \alpha(m+n)$. Thus, $\left|N_{G}[C]\right| \geq \alpha m+(\alpha-1) n$. Hence, $C$ is a connected $(\alpha,(\alpha-1) n)$-partial dominating set in $G$, so Condition ( $a$ ) holds. Similarly, if Condition (ii) holds, then Condition $(b)$ is true. Finally, Condition (iii) is the same as Condition $(c)$.

For the converse, suppose that Condition (a) holds. Then $C \subseteq V(G)$ and $C$ is an $(\alpha,(\alpha-1) n)$-partial dominating set in $G$ by Remark 7. Thus, by Theorem $3, C \subseteq V(G+H)$ is an $\alpha$-partial dominating set in $G+H$. Moreover, $C$ is connected in $G+H$ since $C$ is connected in $G$. Thus, $C$ is a connected $\alpha$-partial dominating set in $G+H$. Similarly, if Condition ( $b$ ) holds, it follows that $C$ is a connected $\alpha$-partial dominating set in $G+H$. If Condition (c) holds, then by Theorem $3, C \subseteq V(G+H)$ is an $\alpha$-partial dominating set in $G+H$. Moreover, $C$ is clearly a connected $\alpha$-partial dominating set in $G+H$.

Corollary 2. Let $G$ and $H$ be connected graphs, $\alpha \in(0,1]$ and let $C \subseteq V(G+H)$ satisfying one of the following conditions:

(i) $C \subseteq V(G)$ is a connected $\alpha$-partial dominating set in $G$.

(ii) $C \subseteq V(H)$ is a connected $\alpha$-partial dominating set in $H$.

(iii) $|C \cap V(G)| \geq 1$ and $|C \cap V(H)| \geq 1$.

Then $C$ is a connected $\alpha$-partial dominating set in $G+H$. 
Proof: Let $|V(G)|=m$ and $|V(H)|=n$. Suppose that Condition $(i)$ holds. It follows by Remark 6 that $C$ is a connected $(\alpha, k)$-partial dominating set in $G$ for $k=(\alpha-1) n$. By Theorem 4(a), $C$ is a connected $\alpha$-partial dominating set in $G+H$. Similarly, if Condition (ii) holds, then $C$ is a connected $(\alpha, k)$-partial dominating set in $H$ for $k=(\alpha-1) m$ and the desired conclusion follows. Finally, if Condition (iii) holds, then $C \cap V(G) \neq \varnothing$ and $C \cap V(H) \neq \varnothing$. Thus, $C$ is a connected $\alpha$-partial dominating set in $G+H$ by Theorem $4(\mathrm{c})$.

Corollary 3. Let $G$ and $H$ be connected graphs of orders $m$ and $n$, respectively, and let $\alpha \in(0,1]$. Then,

$$
\partial_{C \alpha}(G+H)= \begin{cases}1, & \text { if } \partial_{\alpha,(\alpha-1) n}(G)=1 \text { or } \partial_{\alpha,(\alpha-1) m}(H)=1 \\ 2, & \text { otherwise. }\end{cases}
$$

Proof: Suppose that $\partial_{\alpha,(\alpha-1) n}(G)=1$. Let $x \in V(G)$ such that $S=\{x\}$ is an $\partial_{\alpha,(\alpha-1) n}$-set in $G$. Then by definition, $\left|N_{G}[S]\right| \geq \alpha m+(\alpha-1) n$. Now, $\left|N_{G+H}[S]\right|=n+\left|N_{G}[S]\right| \geq$ $n+\alpha m+(\alpha-1) n=\alpha(m+n)$, hence $S$ is both an $\partial_{\alpha}$-set and a $\partial_{C \alpha}$-set in $G+H$ and $\partial_{C \alpha}(G+H)=1$. Similarly, if $\partial_{\alpha,(\alpha-1) n}(H)=1$ then $\partial_{C \alpha}(G+H)=1$. Otherwise, pick a $z \in V(G)$ and a $y \in V(H)$. Clearly, $S=\{z, y\}$ is a $\gamma_{c}$-set, hence a $\partial_{C \alpha}$-set, in $G+H$ and thus, $\partial_{C \alpha}(G+H)=2$.

Theorem 5. Let $G$ be a nontrivial connected graph of order $m$ and $H$ be any graph of order $n$. Let $\alpha \in(0,1]$ and $C \subseteq V(G \circ H)$. If $C \subseteq V(G)$ where $\langle C\rangle$ is connected and either $C$ is a dominating set in $G$ and $|C| \geq \frac{\alpha m(n+1)-m}{n}$ or $|C| \geq \alpha m$, then $C$ is a connected $\alpha$-partial dominating set in $G \circ H$.

Proof: Since $\langle C\rangle$ is connected, it remains to show that $C$ is an $\alpha$-partial dominating set in $G \circ H$. Suppose that $C$ is a dominating set in $G$ and $|C| \geq \frac{\alpha m(n+1)-m}{n}$. Then,

$$
\begin{aligned}
\left|N_{G \circ H}[C]\right| & =|C| n+m \\
& \geq \frac{\alpha m(n+1)-m}{n} \cdot n+m \\
& =\alpha m(n+1)-m+m \\
& =\alpha m(n+1) \\
& =\alpha|V(G \circ H)| .
\end{aligned}
$$

Next, suppose that $C \subseteq V(G)$ with $|C| \geq \alpha m$. Then, since $\left|N_{G}[C]\right| \geq|C|$,

$$
\begin{aligned}
\left|N_{G \circ H}[C]\right| & =|C| n+\left|N_{G}[C]\right| \\
& \geq \alpha m n+\alpha m \\
& =\alpha m(n+1)=\alpha|V(G \circ H)| .
\end{aligned}
$$

So, in either case, $C$ is a connected $\alpha$-partial dominating set in $G \circ H$. 
Example 2. Consider the corona $P_{4} \circ P_{4}$ and $P_{4} \circ C_{4}$ in Figure 7. Let $\alpha=\frac{1}{2}$. Let $S_{1}=\{a, b\} \subseteq V\left(P_{4}\right)$ and $S_{2}=\{v, w\} \subseteq V\left(P_{4}\right)$, where $\left\langle S_{1}\right\rangle$ and $\left\langle S_{2}\right\rangle$ are connected. Now, $S_{1}$ is not a dominating set in $P_{4}$ but $\left|S_{1}\right|=2 \geq \alpha m$, while $S_{2}$ is a dominating set in $P_{4}$ and $\left|S_{2}\right|=2 \geq \frac{3}{2}=\frac{\alpha m(n+1)-m}{n}$. That $S_{1}$ and $S_{2}$ are connected $\alpha$-partial dominating sets in $P_{4} \circ P_{4}$ and $P_{4} \circ C_{4}$, respectively, verifies Theorem 5 .

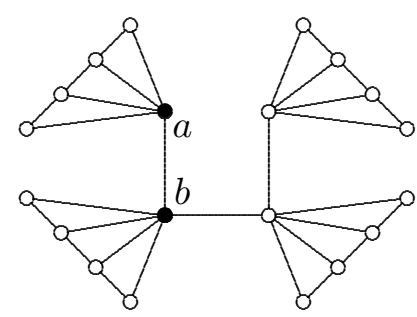

$P_{4} \circ P_{4}$

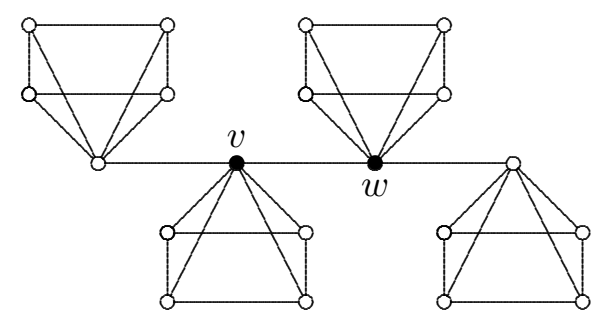

$P_{4} \circ C_{4}$

Figure 7: Connected $\frac{1}{2}$-partial dominating sets in $P_{4} \circ P_{4}$ and $P_{4} \circ C_{4}$, respectively

Remark 8. The converse of Theorem 5 is not true.

To see this, consider the graph $P_{4} \circ H$ where $H=P_{2} \cup P_{1}$ in Figure 8. Let $\alpha=\frac{3}{16}$. The shaded vertices form a connected $\alpha$-partial dominating set in $P_{4} \circ H$ but $C=\left\{u_{1}, u_{2}\right\}$ is not a subset of $V\left(P_{4}\right)$.

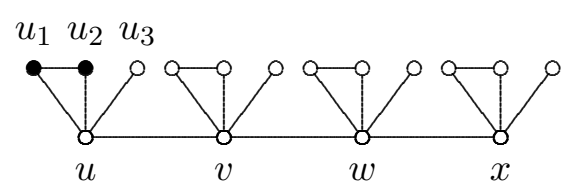

Figure 8: The graph $P_{4} \circ H$ with a connected $\alpha$-partial dominating set $\left\{u_{1}, u_{2}\right\}$, where $\alpha=\frac{3}{16}$

The next result is an immediate consequence of Theorem 5 .

Corollary 4. Let $G$ be a nontrivial connected graph of order $m$ and $H$ be any graph of order $n$. Let $\alpha \in(0,1]$ and $t=\frac{\alpha m(n+1)-m}{n}$. Then

$$
\partial_{C \alpha}(G \circ H) \leq \min \left\{\eta_{G}, \mu_{G}\right\}
$$

where

$$
\eta_{G}=\min \{|C|: C \text { is a connected dominating set in } G \text { with }|C| \geq t\}
$$


and

$$
\mu_{G}=\min \left\{\left|C^{\prime}\right|: C^{\prime} \subseteq V(G) \text { with }\left\langle C^{\prime}\right\rangle \text { connected and }\left|C^{\prime}\right| \geq \alpha m\right\} .
$$

Remark 9. The bound in Corollary 4 is sharp. However, the strict inequality can be attained.

To see this, consider the graphs shown in Figures 8, 9 and 10. The shaded vertices in each graph form an $\partial_{\alpha}$-set for $\alpha=\frac{1}{2}$ and $\alpha=\frac{3}{8}$, respectively. Thus, for $G \circ H$ shown in Figure 9, where $\alpha=\frac{1}{2}, \partial_{C \alpha}(G \circ H)=2=\min \{2,3\}=\min \left\{\eta_{G}, \mu_{G}\right\}=\eta_{G}$, and for $\alpha=\frac{1}{2}$, $\partial_{C \alpha}\left(P_{8} \circ P_{6}\right)=4=\min \{6,4\}=\min \left\{\eta_{G}, \mu_{G}\right\}=\mu_{G}$. However, for the graph shown in Figure 8 , where $\alpha=\frac{3}{8}, \partial_{C \alpha}\left(P_{4} \circ H\right)=1<2=\min \{2,3\}=\min \left\{\eta_{G}, \mu_{G}\right\}$.

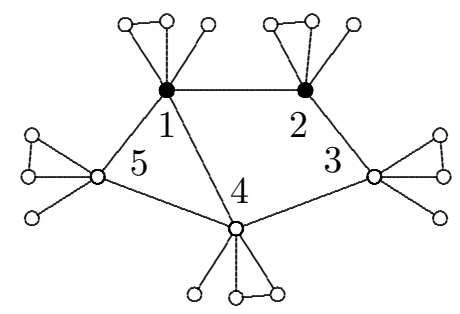

Figure 9: The graph $G \circ H$ with $\partial_{C \alpha}(G \circ H)=2=\min \left\{\eta_{G}, \mu_{G}\right\}=\eta_{G}$, where $\alpha=\frac{1}{2}$

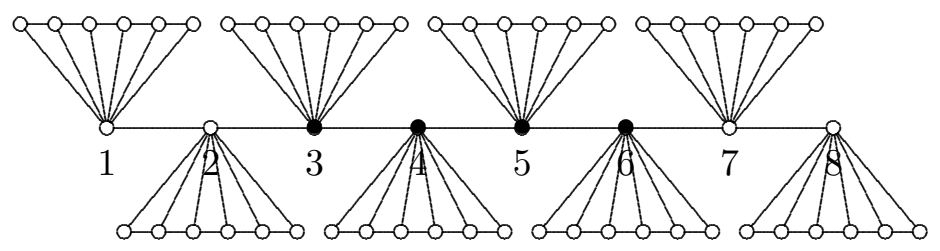

Figure 10: The graph $P_{8} \circ P_{6}$ with $\partial_{C_{\frac{1}{2}}}\left(P_{8} \circ P_{6}\right)=4=\min \left\{\eta_{G}, \mu_{G}\right\}=\mu_{G}$

Lemma 1. Let $\alpha \in(0,1]$ and $C=\bigcup_{x \in S}\left(\{x\} \times T_{x}\right) \subseteq V(G[H])$. Then $\langle C\rangle$ is connected if and only if $\langle S\rangle$ is connected, where $\left\langle T_{x}\right\rangle$ is connected whenever $|S|=1$.

Proof: Suppose that $|S|=1$, say $S=\{x\}$. Assume $\langle C\rangle$ is connected and let $y, z \in T_{x}$ with $y \neq z$. Then there exists an $(x, y)-(x, z)$ geodesic $\left[(x, y)=\left(x_{1}, y_{1}\right),\left(x_{2}, y_{2}\right), \ldots,\left(x_{n}, y_{n}\right)=\right.$ $(x, z)]$ such that $\left(x_{j}, y_{j}\right) \in C$ for all $j$. Since $x_{1}=x_{2}=\ldots=x_{n}=x,\left[y=y_{1}, y_{2}, \ldots, y_{n}=z\right]$ is a $y-z$ geodesic in $H$. Moreover, since $y_{j} \in T_{x}$ for all $j,\left\langle T_{x}\right\rangle$ is connected. Conversely, assume $\left\langle T_{x}\right\rangle$ is connected and let $(x, a),(x, b) \in C$. Then there exists an $a-b$ geodesic $\left[a=a_{1}, a_{2}, \ldots, a_{m}=b\right]$ in $H$, with $a_{j} \in T_{x}$ for all $j$. Consequently, [ $(x, a)=\left(x, a_{1}\right),\left(x, a_{2}\right)$, $\left.\ldots,\left(x, a_{m}\right)=(x, b)\right]$ is an $(x, a)-(x, b)$ geodesic in $G[H]$ with $\left(x, a_{j}\right) \in C$ for all $j$. Hence, $\langle C\rangle$ is connected.

Now supppose that $|S| \geq 2$. Assume $\langle C\rangle$ is connected. Let $x, y \in S$ with $x \neq$ $y$. Pick $z \in T_{x}$ and $w \in T_{y}$. Then there exists an $(x, z)-(y, w)$ geodesic, say $P=$ 
$\left[(x, z)=\left(x_{1}, y_{1}\right), . .,\left(x_{n}, y_{n}\right)=(y, w)\right]$ in $G[H]$ for which $\left(x_{j}, y_{j}\right) \in C$ for all $j$. Since $P$ is a geodesic and $|S| \geq 2,\left[x=x_{1}, x_{2}, \ldots, x_{n}=y\right]$ is a an $x-y$ geodesic in $G$ with $x_{j} \in S$ for all $j$. Hence, $\langle S\rangle$ is connected. Conversely, suppose that $\langle S\rangle$ is connected and let $(x, y),(u, v) \in C$ with $(x, y) \neq(u, v)$. Suppose that $x=u$. Since $|S| \geq 2$ and $\langle S\rangle$ is connected, there exists a $w \in S \backslash\{x\}$ such that $x w \in E(G)$. Pick $z \in T_{w}$. Then $(w, z) \in C$ and $[(x, y),(w, z),(u, v)]$ is a geodesic in $\langle C\rangle$. Suppose that $x \neq u$. Since $\langle S\rangle$ is connected, there exists an $x-u$ geodesic $\left[x=x_{1}, x_{2}, \ldots, x_{n}=u\right]$ in $G$ for which $x_{j} \in S$ for all $j$. Pick, for each $j, y_{j} \in T_{x_{j}}$, where $y_{1}=y$ and $y_{n}=v$. Then $\left[(x, y)=\left(x_{1}, y_{1}\right),\left(x_{2}, y_{2}\right), \ldots,\left(x_{n}, y_{n}\right)=(u, v)\right]$ is an $(x, y)-(u, v)$ geodesic in $G[H]$ with $\left(x_{j}, y_{j}\right) \in C$ for all $j$. Thus, $\langle C\rangle$ is connected.

Theorem 6. Let $G$ and $H$ be nontrivial connected graphs, $\alpha \in(0,1]$, and $C=\bigcup_{x \in S}(\{x\} \times$ $\left.T_{x}\right) \subseteq V(G[H])$.

(i) Suppose that $S=\{x\}$. If $C$ is a connected $\alpha$-partial dominating set in $G[H]$, then $S$ is an $\alpha$-partial dominating set in $G$ and $\left\langle T_{x}\right\rangle$ is connected. Moreover, if $S$ is an $\alpha$-partial dominating set in $G$ and $T_{x}$ is a connected dominating set in $H$, then $C$ is a connected $\alpha$-partial dominating set in $G[H]$.

(ii) Suppose that $|S| \geq 2$. Then $C$ is a connected $\alpha$-partial dominating set in $G[H]$ if and only if $S$ is a connected $\alpha$-partial dominating set in $G$.

Proof: Let $|V(G)|=m$ and $|V(H)|=n$.

(i) Suppose that $C$ is a connected $\alpha$-partial dominating set in $G[H]$. Then, $\left|N_{G[H]}[C]\right|=$ $\left|N_{G}(x)\right| n+\left|N_{H}\left[T_{x}\right]\right| \geq \alpha m n$, thus $\left|N_{G}(x)\right| n \geq \alpha m n-\left|N_{H}\left[T_{x}\right]\right| \geq \alpha m n-n$. Hence, $\left|N_{G}(x)\right| \geq \alpha m-1$, thus $\left|N_{G}[S]\right|=\left|N_{G}[x]\right| \geq(\alpha m-1)+1$, so $\left|N_{G}[S]\right| \geq \alpha m$. Thus, $S$ is an $\alpha$-partial dominating set in $G$. Furthermore, $\left\langle T_{x}\right\rangle$ is connected by Lemma 1. Next, suppose that $S$ is an $\alpha$-partial dominating set in $G$ and $T_{x}$ is a connected dominating set in $H$. Then,

$$
\begin{aligned}
\left|N_{G[H]}[C]\right| & =\left|N_{G}(x)\right| n+\left|N_{H}\left[T_{x}\right]\right| \\
& =\left(\left|N_{G}[x]\right|-1\right) n+\left|N_{H}\left[T_{x}\right]\right| \\
& =\left|N_{G}[S]\right| n-n+n\left(\text { since } T_{x}\right. \text { is a dominating set) } \\
& \geq \alpha m n(\text { since } S \text { is an } \alpha \text {-partial dominating set in } G) .
\end{aligned}
$$

Hence, $C$ is an $\alpha$-partial dominating set in $G[H]$. Furthermore, $C$ is connected by Lemma 1.

(ii) Suppose that $C$ is a connected $\alpha$-partial dominating set in $G[H]$. We claim that $N_{G[H]}[C]=N_{G}[S] \times V(H)$. Let $(x, y) \in N_{G[H]}(C)$. Then either $(x, y) \in N_{G[H]}(C)$ or $(x, y) \in C$. Now,

$$
\begin{aligned}
(x, y) \in N_{G[H]}(C) & \Longrightarrow \exists(u, v) \in C \text { for which }(u, v)(x, y) \in E(G[H]) \\
& \Longrightarrow u \in S \text { and } x \in N_{G}[S]
\end{aligned}
$$




$$
\Longrightarrow(x, y) \in N_{G}[S] \times V(H)
$$

and

$$
\begin{aligned}
(x, y) \in C & \Longrightarrow x \in S \subseteq N_{G}[S] \\
& \Longrightarrow(x, y) \in N_{G}[S] \times V(H) .
\end{aligned}
$$

Conversely, let $x \in N_{G}[S]$ and $y \in V(H)$. Since $|S| \geq 2$ and $\langle S\rangle$ is connected by Lemma 1 , there exists $u \in S$ for which $u x \in E(G)$. Pick $v \in T_{u}$. Then $(u, v) \in C$ and $(x, y) \in$ $N_{G[H]}[(u, v)] \subseteq N_{G[H]}[C]$. Thus, $N_{G}[S] \times V(H) \subseteq N_{G[H]}[C]$. Hence, $N_{G[H]}[C]=N_{G}[S] \times$ $V(H)$. Then, $\left|N_{G[H]}[C]\right|=\left|N_{G}[S]\right| n \geq \alpha m n$, so $\left|N_{G}[S]\right| \geq \alpha m$. Hence, $S$ is an $\alpha$-partial dominating set in $G$ and $\langle S\rangle$ is connected by Lemma 1 .

For the converse, suppose that $S$ is a connected $\alpha$-partial dominating set in $G$. Then $\langle C\rangle$ is connected by Lemma 1 . Moreover, $\left|N_{G[H]}[C]\right|=\left|N_{G}[S]\right| n \geq \alpha m n$, thus $C$ is an $\alpha$-partial dominating set in $G[H]$.

Remark 10. The converse of each of the two statements in Theorem 6(i) is not true.

To see this, consider the graphs $P_{6}\left[P_{7}\right]$ and $P_{5}\left[P_{4}\right]$ in Figure 11 and Figure 12, respectively. Let $\alpha=\frac{1}{2} . S=\{x\}$ is an $\alpha$-partial dominating set in $P_{6}$ and $\left\langle T_{x}\right\rangle$ is connected, but $C=\{(x, a),(x, b),(x, c),(x, d)\}$ does not form an $\alpha$-partial dominating set in $P_{6}\left[P_{7}\right]$ since $\left|N_{P_{6}\left[P_{7}\right]}[C]\right|=20<21=\alpha\left|V\left(P_{6}\right)\right| \cdot\left|V\left(P_{7}\right)\right|$. Moreover, the set $C^{\prime}=\{(x, u)\}$ forms a connected $\alpha$-partial dominating set in $P_{5}\left[P_{4}\right]$ since $\left|N_{P_{5}\left[P_{4}\right]}\left[C^{\prime}\right]\right|=11>10=\alpha\left|V\left(P_{5}\right)\right| \cdot\left|V\left(P_{4}\right)\right|$. However, $T_{x}$ is not a dominating set in $H$.

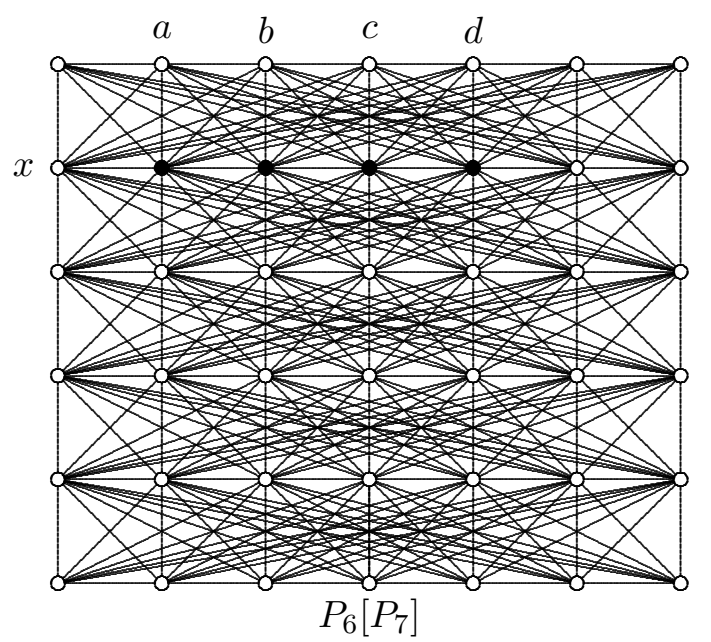

Figure 11: The graph $P_{6}\left[P_{7}\right]$ with $S=\{x\}$ an $\alpha$-partial dominating set in $P_{6}$ and $T_{x}$ connected, where $\alpha=\frac{1}{2}$ 


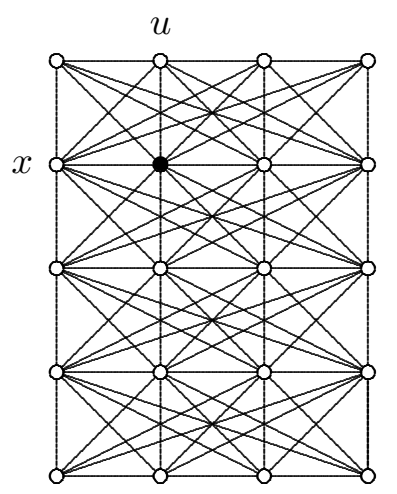

Figure 12: The graph $P_{5}\left[P_{4}\right]$ with $\alpha$-partial dominating set $\{(x, u)\}$, where $\alpha=\frac{1}{2}$

Corollary 5. Let $G$ and $H$ be nontrivial connected graphs and let $\alpha \in(0,1]$. If $\partial_{\alpha}(G)=1$, then $1 \leq \partial_{C \alpha}(G[H]) \leq 2$. If $\partial_{C \alpha}(G) \geq 2$, then $\partial_{C \alpha}(G[H])=\partial_{C \alpha}(G)$.

Proof: Suppose that $\partial_{\alpha}(G)=1$, say $S=\{x\}$ is an $\alpha$-partial dominating set in $G$. Pick $(x, u),(y, v) \in V(G[H])$ such that $x \neq y$ and $x y \in E(G)$. Let $C=\{(x, u),(y, v)\}$. Then $\langle C\rangle$ is connected and

$$
\begin{aligned}
\left|N_{G[H]}[C]\right| & =\left|N_{G[H]}[(x, u)] \cup N_{G[H]}[(y, v)]\right| \\
& =\left|N_{G}(x)\right| \cdot n+\left|N_{G}(y)\right| \cdot n \\
& \geq\left|N_{G}[x]\right| \cdot n\left(\text { since } x \in N_{G}(y)\right) \\
& =\left|N_{G}[S]\right| \cdot n \\
& \geq \alpha m n,
\end{aligned}
$$

hence $C$ is a connected $\alpha$-partial dominating set in $G[H]$. Thus, $\partial_{C \alpha}(G[H]) \leq|C|=2$. Moreover, $\partial_{C \alpha}(G[H]) \geq 1$ by Remark 5 .

Suppose that $\partial_{C \alpha}(G) \geq 2$. Let $C=\bigcup_{x \in S}\left(\{x\} \times T_{x}\right) \subseteq V(G[H])$. Then $|S| \geq 2$, where $S$ is a connected $\alpha$-partial dominating set in $G$. It immediately follows from Theorem $6(i i)$ that $\partial_{C \alpha}(G[H])=\partial_{C \alpha}(G)$.

Remark 11. The bounds given in Corollary 5 for $\partial_{C \alpha}(G[H])$ when $\partial_{\alpha}(G)=1$ are sharp.

To see this, consider the graphs $P_{6}\left[P_{7}\right]$ and $P_{5}\left[P_{4}\right]$ in Figure 11 and Figure 12, respectively. Let $\alpha=\frac{1}{2}$. Note that $\partial_{\alpha}\left(P_{6}\right)=\partial_{\alpha}\left(P_{5}\right)=1$. It can easily be verified that $\partial_{C \alpha}\left(P_{6}\left[P_{7}\right]\right)=2$ and $\partial_{C \alpha}\left(P_{5}\left[P_{4}\right]\right)=1$.

Theorem 7. Let $G$ and $H$ be nontrivial connected graphs and $\alpha \in(0,1]$. Then $C_{1}=$ $S_{1} \times V(H)$ and $C_{2}=V(G) \times S_{2}$ are connected $\alpha$-partial dominating sets in $G \square H$ if and only if $S_{1}$ and $S_{2}$ are connected $\alpha$-partial dominating sets in $G$ and $H$, respectively.

Proof: Let $|V(G)|=m$ and $|V(H)|=n$. Suppose that $C_{1}=S_{1} \times V(H)$ is a connected $\alpha$-partial dominating set in $G \square H$. Then $\left|N_{G \square H}\left[C_{1}\right]\right|=\left|N_{G}\left[S_{1}\right]\right| n \geq \alpha m n$. It follows that 
$\left|N_{G}\left[S_{1}\right]\right| \geq \alpha m$. Hence, $S_{1}$ is an $\alpha$-partial dominating set in $G$. If $\left|S_{1}\right|=1$, then $S_{1}=\{x\}$ for some $x \in V(G)$ and $\left\langle S_{1}\right\rangle$ is connected in $G$. Suppose that $\left|S_{1}\right| \geq 2$. Let $x, y \in S_{1}$ where $x \neq y$ and $x y \notin E(G)$. Pick $a, b \in V(H)$; then $(x, a),(y, b) \in C_{1}$. Since $H$ is connected, there is a geodesic $\left[a=a_{1}, a_{2}, a_{3}, \ldots, a_{m}=b\right]$ from $a$ to $b$ in $H$, and since $\langle C\rangle$ is connected, there is an $(x, a)-(y, b)$ geodesic $\left[(x, a)=\left(x_{1}, a\right),\left(x_{2}, a\right), \ldots,\left(x_{r}, a\right)=\left(y, a_{1}\right), \ldots,\left(y, a_{m}\right)=\right.$ $(y, b)]$ in $G \square H$, where $\left(x_{i}, a\right),\left(y, a_{j}\right) \in C_{1}$ for all $i$ and $j$. It follows that $\left[x=x_{1}, x_{2}\right.$, $\left.\ldots, x_{r}=y\right]$ is a path from $x$ to $y$ in $G$, where $x_{i} \in S_{1}$ for all $i$. Hence, $\left\langle S_{1}\right\rangle$ is connected, thus $S_{1}$ is a connected $\alpha$-partial dominating set in $G$. Similarly, if $C_{2}=V(G) \times S_{2}$ is an $\alpha$-partial dominating set in $G \square H$, then $S_{2}$ is a connected $\alpha$-partial dominating set in $H$.

For the converse, suppose that $C_{1}=S_{1} \times V(H) \subseteq V(G \square H)$ where $S_{1}$ is a connected $\alpha$-partial dominating set in $G$. Then $\left|N_{G \square H}\left[C_{1}\right]\right|=\left|N_{G}\left[S_{1}\right]\right| n \geq \alpha m n$, hence $C_{1}$ is an $\alpha$-partial dominating set in $G \square H$. Suppose that $\left|S_{1}\right|=1$, say $S_{1}=\{x\}$ for some $x \in V(G)$. Let $a, b \in V(H)$, where $a \neq b$. Then $(x, a),(x, b) \in C_{1}$ and $(x, a) \neq(x, b)$. If $a b \in E(H)$, then $(x, a)(x, b) \in E(G \square H)$. If $a b \notin E(H)$, then since $H$ is connected, there is an $a-b$ geodesic $\left[a=a_{1}, a_{2}, \ldots, a_{r}=b\right]$ in $H$. Consequently, $\left[(x, a)=\left(x, a_{1}\right),\left(x, a_{2}\right), \ldots,\left(x, a_{r}\right)=(x, b)\right]$ is an $(x, a)-(y, b)$ geodesic in $G \square H$ with $\left(x, a_{i}\right) \in$ $C_{1}$ for all $i$. Hence, $\left\langle C_{1}\right\rangle$ is connected. Next, suppose that $\left|S_{1}\right| \geq 2$. Let $(x, a),(y, b) \in C_{1}$ with $(x, a) \neq(y, b)$. If $x=y$, then $a \neq b$. From the preceding argument, there is an $(x, a)$ $(y, b)$ geodesic in $\left\langle C_{1}\right\rangle$. Assume that $x \neq y$. Since $\left\langle S_{1}\right\rangle$ is connected, there is an $x$-y geodesic $\left[x=x_{1}, x_{2}, \ldots, x_{r}=y\right]$ in $G$, with $x_{i} \in V(G)$ for all $i$. Since $H$ is connected, there is an $a-b$ geodesic $\left[a=b_{1}, b_{2} \ldots, b_{s}=b\right]$ in $H$. It follows that $\left[(x, a)=\left(x_{1}, b_{1}\right),\left(x_{2}, b_{1}\right), \ldots,\left(x_{r}, b_{1}\right),\left(x_{r}\right.\right.$, $\left.\left.b_{2}\right), \ldots,\left(x_{r}, b_{s}\right)=(y, b)\right]$ is a path from $(x, a)$ to $(y, b)$ in $G \square H$, with $\left(x_{i}, b_{j}\right) \in C_{1}$ for all $i$ and $j$. Hence, $C_{1}$ is a connected $\alpha$-partial dominating set in $G \square H$. Similarly, if $C_{2}=V(G) \times S_{2} \subseteq V(G \square H)$ where $S_{2}$ is a connected $\alpha$-partial dominating set in $H$, then $C_{2}$ is a connected $\alpha$-partial dominating set in $G \square H$.

The next result immediately follows from Theorem 7 .

Corollary 6. Let $G$ and $H$ be nontrivial connected graphs and $\alpha \in(0,1]$. Then, $\partial_{C \alpha}(G \square H) \leq \min \left\{|V(G)| \cdot \partial_{C \alpha}(H),|V(H)| \cdot \partial_{C \alpha}(G)\right\}$.

Remark 12. The bound in Corollary 6 is sharp. However, the strict inequality can be attained.

To see this, consider the graphs shown in Figures 13 and 14. The shaded vertices in each graph form a $\partial_{C \alpha}$-set, where $\alpha=\frac{1}{2}$. Thus,

$$
\begin{gathered}
\partial_{C \alpha}\left(P_{4} \square P_{6}\right)=4=\min \{4 \cdot 1,6 \cdot 1\}=\min \left\{\left|V\left(P_{4}\right)\right| \cdot \partial_{C \alpha}\left(P_{6}\right),\left|V\left(P_{6}\right)\right| \cdot \partial_{C \alpha}\left(P_{4}\right)\right\}= \\
\left|V\left(P_{4}\right)\right| \cdot \partial_{C \alpha}\left(P_{6}\right), \partial_{C \alpha}\left(P_{6} \square P_{3}\right)=3=\min \{6 \cdot 1,3 \cdot 1\}= \\
\min \left\{\left|V\left(P_{6}\right)\right| \cdot \partial_{C \alpha}\left(P_{3}\right),\left|V\left(P_{3}\right)\right| \cdot \partial_{C \alpha}\left(P_{6}\right)\right\}=\left|V\left(P_{3}\right)\right| \cdot \partial_{C \alpha}\left(P_{6}\right), \text { and } \\
\partial_{C \alpha}\left(P_{7} \square P_{8}\right)=10<\{7 \cdot 2,8 \cdot 2\}=\min \left\{\left|V\left(P_{7}\right)\right| \cdot \partial_{C \alpha}\left(P_{8}\right),\left|V\left(P_{8}\right)\right| \cdot \partial_{C \alpha}\left(P_{7}\right)\right\} .
\end{gathered}
$$




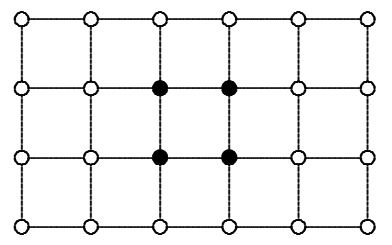

$P_{4} \square P_{6}$

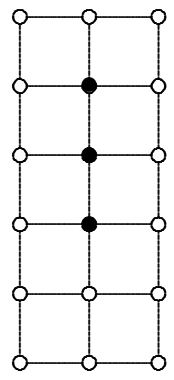

$P_{6} \square P_{3}$

Figure 13: The graphs $P_{4} \square P_{6}$ and $P_{6} \square P_{3}$ with $\partial_{C \alpha}\left(P_{4} \square P_{6}\right)=4$ and $\partial_{C \alpha}\left(P_{6} \square P_{3}\right)=3$, where $\alpha=\frac{1}{2}$

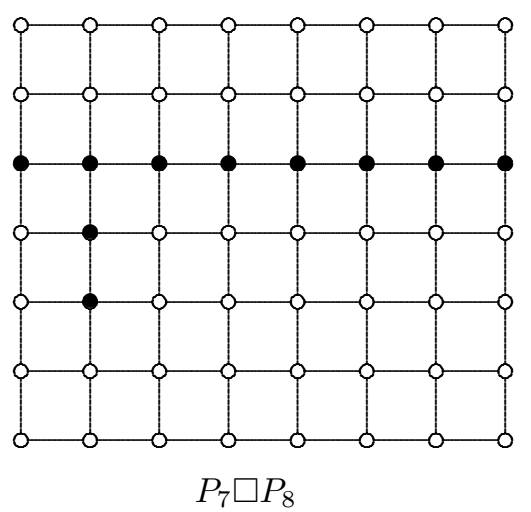

Figure 14: The graph $P_{7} \square P_{8}$ with $\partial_{C \alpha}\left(P_{7} \square P_{8}\right)=10$, where $\alpha=\frac{1}{2}$

\section{Acknowledgements}

This research is funded by the Philippine Department of Science and Technology Accelerated Science and Technology Human Resource Development Program (DOSTASTHRDP), and the MSU-Iligan Institute of Technology. The authors would like to thank the reviewers for their invaluable comments and suggestions. 


\section{References}

[1] A. Behzad. M. Bezhad and C. Praeger. Fundamental dominations in graphs. arXiv: Combinatorics, 2008.

[2] Y. Caro and A. Hansberg. Partial domination - the isolation number of a graph. Filomat, 31(12):3925-3944, 2017.

[3] A. Das. Partial domination in graphs. arXiv: Combinatorics, 2017.

[4] W. Duckworth and B. Mans. Connected domination of regular graphs. Discrete Mathematics, 309(8):2305-2322, 2009.

[5] S. Kaspar. B. Gayathri, M.P. Kulandaivel, and N. Shobanadevi. Towards connected domination in graphs. International Journal of Pure and Applied Mathematics, 117(14):53-62, 2017.

[6] T. Haynes. S. Hedetneimi and P. Slater. Fundamentals of Domination in Graphs. Marcel Dekker, New York, 1998.

[7] R. Macapodi. R. Isla and S. R. Canoy Jr. Partial domination in the join, corona, lexicographic and cartesian products of graphs. Advances and Applications in Discrete Mathematics, 20:277-293, 2019.

[8] H. Karami. S.M. Sheikholeslami. A. Khodkar and D. West. Connected domination number of a graph and its complement. Graphs and Combinatorics, 28:123-131, 2012.

[9] B. M. Case. S.T. Hedetniemi. R.C. Laskar and D.J. Lipman. Partial domination in graphs. arXiv: Combinatorics, 2017.

[10] R. Macapodi and R. Isla. Total partial domination in graphs under some binary operations. European Journal of Pure and Applied Mathematics, 12(4):1643-1655, 2019.

[11] G. Mahalingam. Connected domination in graphs. PhD thesis, University of South Florida, 2005.

[12] E. Sampathkumar and H.B. Walikar. The connected domination number of graphs. Jour. Math. Phy. Sci., 13(6):607-613, 1979.

[13] J. Blum. M. Ding. A. Thaeler and X. Cheng. Connected dominating set in sensor networks and manets. In D. Du and P. Pardalos(eds), editors, Handbook of Combinatorial Optimization. Springer, Boston, MA, 2004. 